Pacific Journal of Mathematics

NATURALLY TOTALLY ORDERED SEMIGROUPS 


\title{
NATURALLY TOTALLY ORDERED SEMIGROUPS
}

\author{
M. SATYanarayana
}

\begin{abstract}
Naturally totally ordered semigroups which are 0 Archimedean are completely characterized by Hölder and Clifford. The purpose of this note is to provide a complete characterization and thus a construction of a class of onesided naturally totally ordered semigroups, which are not o-Archimedean.
\end{abstract}

A totally ordered (t.o.) semigroup $S$ is positively ordered if $a b \geqq a$ and $a b \geqq b$ for all $a, b$ in $S$. A positively totally ordered semigroup $S$ is said to be right (left) naturally totally ordered (n.t.o.) if $a<b$ implies that $b=a x(b=y a)$ for some $x, y \in S$. A right and left n.t.o. semigroup is a n.t.o. semigroup, as defined in [1; 154]. Clifford and Hölder completely characterized order Archimedean (o-Archimedean) n.t.o. semigroups. In [4] the study of onesided n.t.o. semigroups has been initiated. It is observed that right n.t.o. semigroups which are o-Archimedean are n.t.o. Hence their structure is known. In this paper we provide a construction of a class of right n.t.o. semigroups which are not $o$-Archimedean. This is obtained by using the ideas in the proof of Tamura's theorem [3; 55]. We refer the reader to [1] for all the undefined terms mentioned in this paper. An element $x$ in $S$ is called $o$-Archimedean if, for every $y \in S$, there exists an integer $n$ such that $x^{n} \geqq y$. An ideal $P$ in $S$ is called completely prime if, for all $a, b$ in $S, a b \in P$, implies that either $a \in P$ or $b \in P$. An ideal $Q$ in $S$ is called prime if, for all right (two-sided) ideals $A$ and $B$ in $S, A B \subseteq P$ implies that either $A \subseteq P$ or $B \subseteq P$. Clearly every completely prime ideal is prime. We denote the intersection of all prime ideals by $P^{*}$. An element in a semigroup $S$ is said to be central if it commutes with every element in $S$.

LEMMA 1. Let every right ideal in a semigroup $S$ contain an ideal. If $a \in P^{*}$, then for every $x \in S$, there exists a positive integer $m$ such that $a^{m} \in x S$.

Proof. Suppose no power of $a$ is in $x S$. Since $x S$ contains an ideal, say $T$, no power of $a$ is in $T$. Then the collection $P$ of all ideals containing $T$ and not containing any power of $a$ is nonempty. So by Zorn's lemma, there exists an ideal $P$, which is a maximal element in $P$. Now we claim that $P$ is a prime ideal, which establishes the conclusion. Suppose there exist ideals $B$ and $C$ such 
that $B C \subseteq P$, without $B$ and $C$ being contained in $P$. By maximality of $P$, there exist natural numbers $m$ and $n$ such that $a^{m} \in P \cup B$ and $a^{n} \in P \cup C$, so that $a^{m+n} \in P$, which is not true.

Lemma 2. Let $S$ be a semigroup in which every right ideal contains an ideal. Suppose $P^{*}$ contains a central cancellable element $a$ such that $\bigcap_{n=1}^{\infty} a^{n} S=\square$ and $a \notin a S$. If $N$ is the additive semigroup of nonnegative integers; $G$ is a group and $I: G \times G \rightarrow N$ be a function satisfying:

(i) $I(\alpha, \beta)+I(\alpha \beta, \gamma)=I(\alpha, \beta \gamma)+I(\beta, \gamma)$, where $\alpha, \beta, \gamma$ are the elements in $G$.

(ii) $I(\varepsilon, \varepsilon)=1$, where $\varepsilon$ is the identity of $G$ (these two conditions also imply $I(\alpha, \varepsilon)=I(\varepsilon, \alpha)=1$ for every $\alpha \in G)$ then $S$ isomorphic with the semigroup $N \times G$, where the multiplication in $N \times G$ being defined by:

$$
(m, \alpha)(n, \beta)=(m+n+I(\alpha, \beta), \alpha \beta) .
$$

Proof. Essentially the proof is similar to Tamura's proof $[3 ; 56]$ except for some minor changes. We sketch now the important facts. Set $T_{n}=a^{n} S \backslash a^{n+1} S, n=1,2, \cdots$ and $T_{0}=S \backslash a S$. Since $a$ is a central element, every element in $S$ can be written uniquely as $a^{n} z, z \notin a S$. Define a relation $\sigma$ on $S$ by: $x \sigma y$ iff $a^{m} x=a^{n} y$ for some $m, n \geqq 0$. Since $a$ is central, $\sigma$ is a congruence. Denote the classes of $\sigma$ by $S_{\alpha}$, where $\alpha \in G . G$ is a semigroup by defining that the product $\alpha \beta$ in $G$ is the index of the class containing $x y$, where $x \in S_{\alpha}$ and $y \in S_{\beta}$. All positive powers of $a$ constitute a single $\sigma$-class. For, if $x \sigma a$ and if $x$ is not a power of $a$, then $x=a^{m} z, z \notin a S$. Now $a^{p}\left(a^{m} z\right)=a^{q}(a)$ for some $p, q \geqq 0$. If $p+m \leqq q$, then $z=a^{q-p-m+1}$ since $a$ is cancellable and so $x$ is a power of $a$, which is not true. If $p+m>q$, then $a \in a S$, which is again not true. Denote the class containing of all positive powers of $a$ by $S_{\varepsilon}$. Then $\varepsilon$ is the identity of $G$. We observe that $G$ is a group. For, let $\alpha \in G$. If $x \in S_{\alpha}$, they by Lemma $1, a^{m}=x y$ for some natural number $m$ and for some $y$ in $S$. So $a \sigma x y$ and thus if $S_{\beta}$ is the $\sigma$-class containing $y$, then $S_{\varepsilon}=S_{\alpha} S_{\beta}$ and $\varepsilon=\alpha \beta$. By the same reason $\varepsilon=\beta \gamma$ for some $\gamma \in S$. Thus $\varepsilon=\alpha \beta=\beta \alpha$ and hence $G$ is a group. The rest of the proof is similar to that given in the above reference.

The following lemma is of independent interest. This proves the converse of Lemma 2 under some additional hypotheses. The two lemmas do provide structure theorem when the semigroup contains proper prime ideals, which is not the case with Tamura's theorem and cancellative condition is obtained as a consequence. This supplements Nordhal's result [2]. 
Lemma 3. Let $T=N \times G$ be the semigroup as described in Lemma 2, and let $I$ satisfy the additional property that there exists an $\alpha \in G$ such that $I\left(\alpha^{n}, \alpha\right)=0$ for all $n$. Then every right ideal in $T$ contains an ideal and $P^{*}$, which is the intersection of all prime ideals in $T$, contains a central cancellable element a such that $\bigcap_{n=1}^{\infty} a^{n} T=\square$ and $a \notin a T$.

Proof. As noted in [3; 55] the multiplication defined in Lemma 2 is associative. By direct verification one can show that $(0, \varepsilon)$ is a central cancellable element; $\bigcap_{n=1}^{\infty}(0, \varepsilon)^{n} T=\square$ and $(0, \varepsilon) \in(0, \varepsilon) T$. For the rest of the proof we need the following result:

(*) for any $(n, \beta) \in N \times G$, there exists an $m$ such that $(0, \varepsilon)^{m} \in(n, \beta) T$.

This is true because we can choose positive integers $m$ and $k$ such that $m>n+I\left(\beta, \beta^{-1}\right)$ and $k=m-1-n-I\left(\beta, \beta^{-1}\right)$ and hence $(0, \varepsilon)^{m}=(n, \beta)\left(k, \beta^{-1}\right)$.

Now let $A$ be a right ideal. By $(*),(0, \varepsilon)^{m}=(m-1, \varepsilon) \in A$ for some positive integer $m$. Clearly $(0, \varepsilon)^{m}=(m-1, \varepsilon)$ is central. Thus $A$ contains a two-sided ideal $T(m-1, \varepsilon) T$.

We claim now that $T$ has proper prime ideals. By hypothesis there exists an $\alpha$ such that $I\left(\alpha^{n}, \alpha\right)=0$ for all $n$. It can be verified easily $(0, \alpha)^{n} \notin(1, \alpha) T$ for every $n$. But $(1, \alpha) T$ contains an ideal $Q$ from above. So $(0, \alpha)^{n} \notin Q$ for every $n$. Then by Zorn's lemma the nonempty collection of ideals containing $Q$ and not containing any power of $(0, \alpha)$ has a maximal element $P$. We prove now that $P$ is a prime ideal. For, let $A$ and $B$ be ideals such that $A B \leqq P, A \nsubseteq P$, and $B \nsubseteq P$. Then by maximality of $P$ there exist integers $m$ and $n$ such that $(0, \alpha)^{m} \in P \cup A$ and $(0, \alpha)^{n} \in P \cup B$, so that $(0, \alpha)^{m+n} \in P$, which is not true.

Finally we shall show that $(0, \varepsilon)$ belongs to every prime ideal. Let $P$ be a prime ideal and $x \in P$. By (*) there exists an integer $m$ such that $(0, \varepsilon)^{m} \in x S \subseteq P$. Since $(0, \varepsilon)$ is central, $(0, \varepsilon)^{m-1} S^{1}(0, \varepsilon) S^{1} \cong$ $(0, \varepsilon)^{m} S^{1} \subseteq P$. So $(0, \varepsilon)^{m-1} \in P$. Continuing in this manner we have $(0, \varepsilon) \in P$.

LEMMA 4. Let $S$ be a positively t.o. semigroup. Then the set $A$ of all o-Archimedean elements is a completely prime ideal. If $S$ is right n.t.o., then $A$ is included in every completely prime ideal if $A \neq S$.

Proof. It is easy to check that $A$ is an ideal. To prove that $A$ is a completely prime ideal, assume $a b \in A$ with $a$ and $b \notin A$. We may set $a \leqq b$. Then $a b \leqq b^{2}$ and $b^{m}<z$ for some $z$ and for every 
m. This implies $(a b)^{n} \leqq b^{2 n}<z$ for every $n$, which is not true. Suppose now that $P$ is a completely prime ideal in a right n.t.o. semigroup $S$. Let $x \in A$ and $y \in P$. Then there exists an $n$ such that $x^{n} \geqq y$. If $y=x^{n}, x \in P$ since $P$ is completely prime. If $y<x^{n}$, then $x^{n} \in y S \subseteq P$, so that $x \in P$, as before.

LEMMA 5. Let $S$ be a right n.t.o. semigroup. Then every right ideal is an ideal and every prime ideal is completely prime.

Proof. Let $A$ be a right ideal. If $a \in A$ and $s \in S$, then $a \leqq s a$ by positive order. If $a=s a$, then $s a \in A$. If $a<s a$, by right n.t.o. condition, for some $t$ we have $s a=a t$ and so $s a \in A$. Thus $A$ is an ideal. Suppose now that $P$ is a prime ideal and $a b \in P$. Then from the above,

$$
\left(a S^{1}\right)\left(b S^{1}\right) \subseteq a b S^{1} \cup a b S \cup a S b S \cong a b S^{1} \cup a b S \cup a b S^{2} \subseteq P,
$$

which implies $a$ or $b$ belongs to $P$.

THEOREM. Let $N$ be the set of all nonnegative integers and $G$ be a group. Suppose $I$ is a function from $G \times G \rightarrow\{0,1\}$ satisfying the following properties: G.

(i ) $I(\alpha, \beta)+I(\alpha \beta, \gamma)=I(\alpha, \beta \gamma)+I(\beta, \gamma)$ for every $\alpha, \beta, \gamma$ in

(ii) $I(\varepsilon, \varepsilon)=1, \varepsilon$ being the identity of $G$.

(iii) There exists a nonperiodic element $\alpha \in G$ such that for all but a finite number of $n, I\left(\alpha^{n}, \alpha\right)=0$.

(iv) $I\left(\alpha, \alpha^{-1}\right)=0$ for every $\alpha \neq \varepsilon$ in $G$.

(v) For $\alpha \neq \beta, \alpha \neq \varepsilon, \beta \neq \varepsilon$ one of $I\left(\beta, \beta^{-1} \alpha\right)$ and $I\left(\alpha, \alpha^{-1} \beta\right)$ is zero and the other is 1 .

(vi) If $\left(\beta, \beta^{-1} \alpha\right)=0$, then for every $\gamma$ we have only the following possibilities:

(a) $I(\beta, \gamma)=0$ and $I(\alpha, \gamma)=1$

(b) $I(\beta, \gamma)=I(\alpha, \gamma)=I\left(\beta \gamma,(\beta \gamma)^{-1} \alpha \gamma\right)=0$

(c) $I(\beta, \gamma)=1=I(\alpha, \gamma)$ and $I\left(\beta \gamma,(\beta \gamma)^{-1} \alpha \gamma\right)=0$.

(vii) If $I\left(\beta, \beta^{-1} \alpha\right)=0$, then for every $\gamma$ we have only the following possibilities:

(a) $I(\gamma, \beta)=0$ and $I(\gamma, \alpha)=1$

(b) $I(\gamma, \beta)=I(\gamma, \alpha)=I\left(\gamma \beta,(\gamma \beta)^{-1} \gamma \alpha\right)=0$

(c) $I(\gamma, \beta)=1=I(\gamma, \alpha)$ and $I\left(\gamma \beta,(\gamma \beta)^{-1} \gamma \alpha\right)=0$.

(viii) If $I(\beta, \gamma)=1$ and $I(\alpha, \gamma)=0$, then $I\left(\beta \gamma,(\beta \gamma)^{-1} \alpha \gamma\right)=0$.

(ix ) If $I(\gamma, \beta)=1$ and $I(\gamma, \alpha)=0$, then $I\left(\gamma \beta,(\gamma \beta)^{-1} \gamma \alpha\right)=0$.

Define multiplication in $N \times G$ by:

$$
(m, \alpha)(n, \beta)=(m+n+I(\alpha, \beta), \alpha \beta) .
$$


Define order in $N \times G$ by: if $m \geqq 1,(m, \alpha)>(0, \beta) ;(0, \varepsilon)>(0, \beta)$ if $\beta \neq \varepsilon ;$ for $\alpha \neq \beta, \alpha \neq \varepsilon, \beta \neq \varepsilon,(0, \alpha)>(0, \beta)$ if $I\left(\beta, \beta^{-1} \alpha\right)=0$; if $m>n,(m, \alpha)>(n, \beta) ;(m, \alpha)>(m, \beta)$ if $I\left(\beta, \beta^{-1} \alpha\right)=0$. Then $N \times G$ is a right n.t.o. semigroup not containing 1 and is non-o-Archimedean but contains a central cancellable o-Archimedean element. Conversely every such semigroup can be constructed in this way.

Proof. By direct verification one can establish that $N \times G$ is a right n.t.o. semigroup not containing 1 . By (iii), there exists an $\alpha$ such that all but a finite number, say $m$ of $I\left(\alpha^{i}, \alpha\right)$ are zero. Then for any $n(0, \alpha)^{n}=\left(t, \alpha^{n}\right)$ and $t<m+1$ since $\alpha$ is not periodic and hence $(0, \alpha)^{n}<(m+1, \varepsilon)$. Therefore $N \times G$ is not $o$-Archimedean. It is easy to verify that $(0, \varepsilon)$ is a central $o$-Archimedean element.

Conversely let $S$ be a right n.t.o. semigroup containing a central cancellable $o$-Archimedean element $a$. Then by Lemmas 4 and $5, P^{*}$ contains $a . a \notin a S$, since otherwise we have for some $s, a=a s=a s^{2}$, so that $s=s^{2}$ by cancellative condition of $a$. Now if $y \in S, y a=$ $y a s=y s a$, which implies $y=y s$. But $(a s) y=a y$. So $(s y) a=y a$ and $s y=y$. Thus $s$ is an identity, which is a contradiction. Hence $a \notin a S$. Also $\bigcap_{n=1}^{\infty} a^{n} S=\square$. For if $y \in \bigcap_{n=1}^{\infty} a^{n} S, y=a^{n} s_{n}$ and so $y \geqq a^{n}$. Since $a$ is 0 -Archimedean, we have $a^{n}=y$. Now $a^{n} \in \bigcap_{n=1}^{\infty} a^{n} S$ implies $a^{n}=a^{n+1}$ by positive order and hence $a=a^{2}$ by cancellation, which is again untrue as before. Combining Lemmas 2, 4, and 5, we have that $S$ is isomorphic with $N \times G$, with the $I$-function satifying the first two properties stated in the theorem. If $\alpha \neq \varepsilon$ and if $(0, \alpha)$ is in $(0, \varepsilon) S$, then we must have $(0, \alpha)=(0, \varepsilon)(0, \alpha)$, which implies $I(\varepsilon, \alpha)=0$, which contradicts the consequence of the properties (i) and (ii), as noted in Lemma 2 . Since $S$ is right n.t.o. we must have then $(0, \varepsilon)$ is in $(0, \alpha) S$, which implies $(0, \varepsilon)=(0, \alpha)$ $\left(0, \alpha^{-1}\right)$ and so $I\left(\alpha, \alpha^{-1}\right)=0$. Now set $\gamma=\beta^{-1}$ in (i). Then if $\beta \neq \varepsilon$,

$$
I(\alpha, \beta)+I\left(\alpha \beta, \beta^{-1}\right)=I(\alpha, \varepsilon)+I\left(\beta, \beta^{-1}\right)=1+0 .
$$

Thus $I(\alpha, \beta)=0$ or 1 and hence $I(\alpha, \beta)=0$ or 1 for every $\beta \in G$. Therefore $I$ maps $G \times G$ into $\{0,1\}$.

To prove (iii), observe that $S$ contains non-o-Archimedian elements. Therefore there exist $(m, \alpha)$ and $(n, \beta)$ in $N \times G$ such that $(m, \alpha)^{r}<(n, \beta)$ for every natural number $r$. If $m \neq 0$ and $n \neq 0$, then there exists an $k$ such that $k m>n$. Then

$$
(m, \alpha)^{k+1}=\left((k+1) m+I(\alpha, \alpha)+\cdots+I\left(\alpha^{k+1}, \alpha\right), \alpha^{k+1}\right)
$$

and hence $(m, \alpha)^{k+1}>(n, \beta)$, which is not true. If $m=0$ and $n \neq 0$, then $(0, \alpha)^{r}<(n, \beta)$ for all $r$ implies $I(\alpha, \alpha)+\cdots+I\left(\alpha^{r}, \alpha\right) \leqq n$ for every natural number $r$. Therefore all but a finite number of 
$I\left(\alpha^{i}, \alpha\right)$ are zero. If $m=n=0$, then it is clear that $I\left(\alpha^{i}, \alpha\right)=0$ for every $i$. The case $m \neq 0$ and $n=0$ is inadmissible. $\alpha$ is not periodic since otherwise if $\alpha^{s}=\varepsilon$, then $(0, \alpha)^{s}=(N, \varepsilon)$ where $N=$ $I(\alpha, \alpha)+\cdots+I\left(\alpha^{s}, \alpha\right)$ and $(0, \alpha)^{t_{s}}=(t(N+1)-1, \varepsilon)$. Choose $k$ such that $k(N+1)-1>n$. Therefore

$$
(0, \alpha)^{k s}=(k(N+1)-1, \varepsilon)>(n, \varepsilon)>(n, \beta),
$$

which is not true. It is routine to check that the remaining conditions are necessary for the admissibility of right n.t.o. structure on $N \times G$.

In commutative case, the $I$-function satisfies the property $I(\alpha, \beta)=I(\beta, \alpha)$, in addition to the above. The commutative example supporting the hypothesis of the theorem is $N \times G$, where $G$ is an infinite cyclic group generated by $x$ and the $I$-function is defined by: $I\left(x^{n}, x^{m}\right)=0$ if $n, m>0 ; I\left(x^{n}, x^{-m}\right)=0$ if $0<n \leqq m$ and in all other cases the value of $I$-function is 1 .

Since $N \times G$ is cancellative, the right n.t.o. semigroup containing central cancellable $o$-Archimedean elements, which is not $o$-Archimedean, is in fact cancellative. By condition (iii) of the theorem, the group $G$ is necessarily nonperiodic and $N \times G$ is not finitely generated since by corollary of $[4 ; 11]$ cancellative finitely generated right n.t.o. semigroups are infinite cyclic semigroups (possibly adjoined with 1) and hence o-Archimedean. More than this we do not know the structure of $G$. So the problem "what groups admit the $I$-function with the prescribed properties in the theorem" remains open. It may be remarked that a particular case of this theorem has been solved by Etterbeck in his thesis, written at the University of California, Davis.

\section{REFERENCES}

1. L. Fuchs, Partially Ordered Algebraic Systems, Pergamon Press, 1963.

2. T. Nordhal, Cancellative semigroups with nonempty center, Semigroup Forum, 11 (1975), 170-177.

3. M. Petrich, Introduction to Semigroups, Charles E. Merrill Publishing Company, 1973.

4. M. Satyanarayana and C. Srihari Nagore, Naturally ordered semigroups (submitted).

Received May 2, 1977 and in revised form November 23, 1977.

Bowling Green State University

Bowling GREEN, OH 43403 


\section{PACIFIC JOURNAL OF MATHEMATICS}

\section{EDITORS}

RICHARD ARENS (Managing Editor)

University of California

Los Angeles, California 90024

C. W. Curtis

University of Oregon

Eugene, OR 97403

C. C. MOORE

University of California

Berkeley, CA 94720

\section{J. DUGUNDJI}

Department of Mathematics University of Southern California Los Angeles, California 90007

R. Finn AND J. Milgram Stanford University Stanford, California 94305

\section{ASSOCIATE EDITORS}

E. F. BeCK ENBACH

B. H. NeUMaNN

F. WOLF

K. Yoshida

\section{SUPPORTING INSTITUTIONS}

UNIVERSITY OF BRITISH COLUMBIA CALIFORNIA INSTITUTE OF TECHNOLOGY UNIVERSITY OF CALIFORNIA MONTANA STATE UNIVERSITY UNIVERSITY OF NEVADA, RENO NEW MEXICO STATE UNIVERSITY OREGON STATE UNIVERSITY UNIVERSITY OF OREGON
UNIVERSITY OF SOUTHERN CALIFORNIA STANFORD UNIVERSITY UNIVERSITY OF HAWAII UNIVERSITY OF TOKYO UNIVERSITY OF UTAH WASHINGTON STATE UNIVERSITY UNIVERSITY OF WASHINGTON 


\section{Pacific Journal of Mathematics \\ Vol. 77, No. $1 \quad$ January, 1978}

Dan Amir, Chebyshev centers and uniform convexity ............... 1

Lawrence Wasson Baggett, Representations of the Mautner group. I ..... 7

George Benke, Trigonometric approximation theory in compact totally

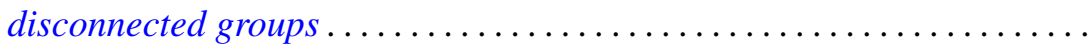

M. Bianchini, O. W. Paques and M. C. Zaine, On the strong compact-ported topology for spaces of holomorphic mappings ..................

Marilyn Breen, Sets with $(d-2)$-dimensional kernels

J. L. Brenner and Allen Kenneth Charnow, Free semigroups of $2 \times 2$

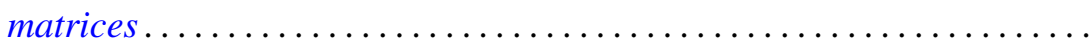

David Bressoud, A new family of partition identities .................

David Fleming Dawson, Summability of matrix transforms of stretchings

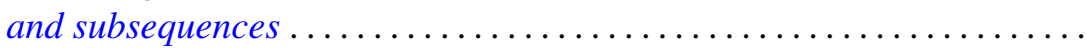

Harold George Diamond and Paul Erdôs, A measure of the nonmonotonicity

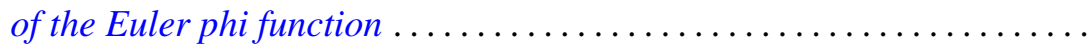

Gary Doyle Faulkner and Ronald Wesley Shonkwiler, Kernel dilation in reproducing kernel Hilbert space and its application to moment

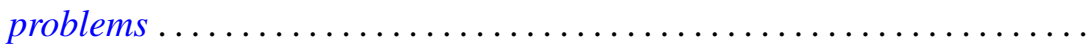

Jan Maksymilian Gronski, Classification of closed sets of attainability in the

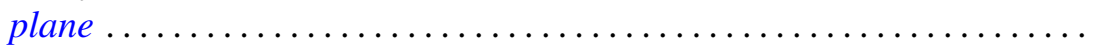

H. B. Hamilton and T. E. Nordahl, Semigroups whose lattice of congruences

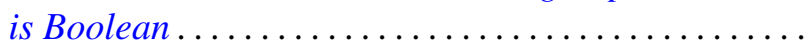

Harvey Bayard Keynes and D. Newton, Minimal $(G, \tau)$-extensions ...

Anthony To-Ming Lau, The Fourier-Stieltjes algebra of a topological

semigroup with involution.

B. C. Oltikar and Luis Ribes, On prosupersolvable groups ...

Brian Lee Peterson, Extensions of pro-affine algebraic groups ...

Thomas M. Phillips, Primitive extensions of Aronszajn spaces ...

Mehdi Radjabalipour, Equivalence of decomposable and 2-decomposable operators. .

M. Satyanarayana, Naturally totally ordered semigroups .

Fred Rex Sinal, A homeomorphism classification of wildly imbedded two-spheres in $S^{3}$

Hugh C. Williams, Some properties of a special set of recurring

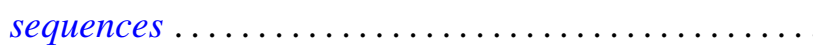

